\title{
Comparative analysis of dietary exosome-derived microRNAs from human, bovine and caprine colostrum and mature milk
}

\author{
Bohyun Yun ${ }^{1}$, Younghoon Kim², Dong June Park ${ }^{3 *}$ and Sangnam Oh${ }^{1 *}$ \\ ${ }^{1}$ Department of Functional Food and Biotechnology, Jeonju University, Jeonju 55069, Korea \\ ${ }^{2}$ Department of Agricultural Biotechnology and Research Institute of Agriculture and Life Science, Seoul \\ National University, Seoul 08826, Korea \\ ${ }^{3}$ Korea Food Research Institute, Wanju 55365, Korea
}

Received: Jan 18, 2021

Revised: Jan 21, 2021

Accepted: Jan 25, 2021

*Corresponding author

Dong June Park

Korea Food Research Institute, Wanju

55365, Korea.

Tel: +82-63-219-9114

E-mail: djpark@kfri.re.kr

Sangnam Oh

Department of Functional Food and

Biotechnology, Jeonju University,

Jeonju 55069, Korea.

Tel: +82-63-220-3109

E-mail: osangnam@jj.ac.kr

Copyright (C) 2021 Korean Society of Animal Sciences and Technology.

This is an Open Access article distributed under the terms of the Creative Commons Attribution

Non-Commercial License (http:// creativecommons.org/licenses/bync/4.0/) which permits unrestricted non-commercial use, distribution, and reproduction in any medium, provided the original work is properly cited.

ORCID

Bohyun Yun

https://orcid.org/0000-0001-6723-5849

Younghoon Kim

https://orcid.org/0000-0001-6769-0657

Dong June Park

https://orcid.org/0000-0001-9452-9391

Sangnam $\mathrm{Oh}$

https://orcid.org/0000-0002-2428-412X

Competing interests

No potential conflict of interest relevant

to this article was reported.

\begin{abstract}
Mammalian milk including microRNAs (miRNAs) as a novel class of noncoding RNAs, that can be transferred to infants and it plays on a critical role in biological functions such as immune regulation and development. However, the origin and functional importance of milk-derived miRNAs are still undetermined. This study applied RNA sequencing to explore the featured profiles of miRNA expression in colostrum and mature milk-originated exosomes from human, bovine, and caprine milk. These dietary exosome-derived miRNAs are highly conserved in human, bovine and caprine milk. Interestingly, abundant miRNAs expressed in human milk are similarly conserved across species. In addition, we confirmed that immune-related miRNAs (miR-30a-5p, miR-22-3p, and miR-26a) are commonly observed in the colostrum and mature milk of cows and caprines as well as humans. Our results provide new insights and resources for investigating the functionality of immune-associated miRNAs and evaluating physiological and biological condition in human, bovine and caprine milk as biomarkers.
\end{abstract}

Keywords: Colostrum, Mature milk, Dietary exosome, MicroRNA, Mammals

\section{INTRODUCTION}

Milk is a major nutritional source for infants, and breastfeeding is one of the most valuable participants to infant health [1]. In addition, breastfeeding reduces the risk of acute infant-related diseases by providing an infant with a developmental program and protection against infection [2-4]. The components of breast milk can contribute the immune response of newborns, and they influence on the development of infant and children's immune systems. However, the mechanisms of immune modulation by breast milk are still unclear and might predicted from the role of biological macromolecules existed in breast milk including antibodies, growth factors, and nucleic acids [5]. An additional unique bioactive component of breast milk recently discovered is microRNA (miRNA) $[6,7]$. 
Funding sources

This research was supported by the High

Value-Added Food Technology Development

Program of the Korean Institute of Planning

and Evaluation for Technology in Food,

Agriculture, Forestry, and Fisheries

(iPET), the Ministry for Food, Agriculture,

Forestry, and Fisheries of the Korea

(318090-03-1-WT011) and the National

Research Foundation of Korea Grant

funded by the Korean government (NRF-

2018R1D1A3B07050304).

Acknowledgements

Not applicable.

Availability of data and material Upon reasonable request, the datasets of this study can be available from the corresponding author.

Authors' contributions

Conceptualization: Yun B, Kim Y, Park DJ,

Oh S.

Data curation: Yun B, Kim Y, Park DJ, Oh S.

Formal analysis: Yun B, Kim Y, Park DJ, Oh S.

Methodology: Yun B, Kim Y, Park DJ, Oh S.

Software: Yun B, Kim Y, Park DJ, Oh S.

Validation: Yun B, Kim Y, Park DJ, Oh S.

Investigation: Yun B, Kim Y, Park DJ, Oh S.

Writing - original draft: Yun B, Kim Y, Park

DJ, Oh S.

Writing - review \& editing: Yun B, Kim Y, Park

DJ, Oh S.

Ethics approval and consent to participate All procedures in this study were approved by the Investigational Review Board (IRB) of Jeonju University (jJIRB-210215HB-2021-0212).
Since 1993 discovered on their importance, it has been well-established that miRNAs composed with 18-22 nucleotides in length as a small class of noncoding RNAs (ncRNAs), principally contribute as post-transcriptional regulators of target gene expression by RNA interference [8]. In particular, they have served as key players of gene regulation at the post-transcriptional level in humans, animals and plants [9-11]. In addition, a variety of miRNAs are present in body fluidic system contacting tears, saliva, plasma, and urine as well as commonly in breast milk [12]. Exosomes are membrane-bound extracellular vesicles (EVs) with a diameter of 40 to $100 \mathrm{~nm}$ that are released from the cells into the extracellular space after being formed within multivesicular bodies in the endosome system [13]. The exosomal cargo is genetic material including DNA, mRNA, miRNA, proteins and lipids [14]. In particular, they are abundant in various type of RNA including coding RNA and ncRNA [8]. Interestingly, previous studies indicated that exosomes are rich in breast milk and contains miRNAs related to the immune system as well as the nutrition of newborns $[8,15,16]$. Exosomes are continuously secreted into mammary fluidic system (e.g., mature milk and colostrum), and they act a key player for intracellular communication by carrying their contents (e.g., miRNA) to target cells $[17,18]$. Initially, existence of milk-derived exosomes were reported from human breast milk [19] and later in milk and colostrum from animals such as cows [20], caprines [21], and pigs [15]. Previous studies have characterized and investigated on a number of miRNAs that originated from milk exosomes of various mammals $[6,8,22]$. However, less information is available on the identification of miRNAs in exosomes derived from milk components present in various mammals. Identification of miRNAs in exosomes isolated from colostrum and mature milk from various mammals can be useful for exploring biological functionality related to the newborn's immune system. The purpose of this study was to identify profiling of miRNAs and characterize their genetic diversities in exosomes derived from the colostrum and mature milk of three different species of mammals.

\section{MATERIALS AND METHODS}

\section{Milk samples}

Bovine colostrum and mature milk samples were collected from 6 healthy Holstein cows (for colostrum, it was taken from the cows within 3 days postpartum, $n=3$; for mature milk, it was taken from cows at 30 days postpartum, $n=3$ ). In the same manner, caprine colostrum and mature milk samples were also obtained from the Saanen breed for colostrum $(\mathrm{n}=3)$ and mature milk ( $\mathrm{n}$ =3). Moreover, human breast milk samples were collected from two volunteers (Jeonju, Jeollabukdo, Korea). Human breast colostrum and mature milk samples were collected from healthy mothers during the first 3 days and 30 days after delivery, respectively. Each milk sample was collected, and then the collected samples totaling $50-100 \mathrm{~mL}$ were put into storage bags. All milk samples were frozen immediately after collection and were kept at $-80^{\circ} \mathrm{C}$ until use. All procedures in this study were approved by the Investigational Review Board (IRB) of Jeonju University (jjIRB-2020-1205).

\section{Isolation of dietary exosome from the milk samples}

Isolation of exosomes from the milk was performed using established methods in previous studies with minor modifications [23]. Briefly, the milk samples were centrifuged at $1,500 \times \mathrm{g}$ and $4{ }^{\circ} \mathrm{C}$ for $30 \mathrm{~min}$ to remove fat, cells, and debris. The supernatant was defatted at $16,500 \times \mathrm{g}$ for $1 \mathrm{~h}$ at $4^{\circ} \mathrm{C}$ to remove the casein. The casein-removed supernatant (whey) was then passed through 0.45 - and $0.22-\mu \mathrm{m}$ filters to remove residual cell debris. The clear supernatant was carefully collected to avoid contamination of the pelletized exosomes. The pelletized exosomes were resuspended in phosphatebuffered saline (PBS), followed by ultracentrifugation for washing $\left(100,000 \times \mathrm{g}, 4^{\circ} \mathrm{C}, 90 \mathrm{~min}\right)$. After 
washing twice with additional ultracentrifugation $\left(135,000 \times \mathrm{g}, 4^{\circ} \mathrm{C}, 90 \mathrm{~min}\right)$, the exosomes were reconstituted in PBS.

\section{Extraction of miRNA and small RNA-sequencing}

Extraction of miRNA from the milk exosomes was performed using established methods in previous studies with minor modifications [24]. Briefly, miRNA was extracted from milk exosomes using combined phenol and column methods: phenol methods using Qiazol ${ }^{\circledR}$ (Qiagen, Hilden, Germany) and column methods using the miRNeasy ${ }^{\circledR}$ Mini kit (miRNeasy I, Qiagen). All methods were employed according to the manufacturer's instruction. The miRNAs extracted were re-suspended with $50 \mu \mathrm{L}$ of nuclease-free water. The integrity of the miRNAs as well as quality and quantity were determined on an Agilent 2100 Bioanalyzer using an RNA 6000 Pico Kit (Agilent Technologies, Santa Clara, CA, USA) according to the manufacturer's manual. The sequencing service and library preparation were provided by Macrogen, Korea. All libraries were sequenced using the HiSeq platform (Illumina).

\section{Bioinformatics analysis}

Low quality reads were removed from the raw data, high quality reads were then subjected to sRNAbench for adapter sequence trimming and length distribution analysis read. The sequences with read length larger than 15 nt were aligned against human, bovine, and caprine miRNA database (miRBase, release ver. 22) [25] with the default parameters to identify known miRNAs using sRNAbench and did not allow any discrepancies. Each library was processed separately, and the expression level of miRNA was estimated by sRNAbench, which normalized the number of reads for each miRNA RPM. The miRNA unique sequences were applied to investigate the level of the miRNA expression based on the normalized read count.

\section{Statistical analysis}

All data are expressed as the means \pm SD. Statistical analysis was performed by one-way analysis of variance (ANOVA). A value of $p<0.05$ was considered statistically significant.

\section{RESULTS AND DISCUSSION}

Comparison of miRNAs in exosomes from human breast colostrum and mature milk Initially, human breast milk was profiled for miRNA using a small RNA sequencing method to compare colostrum collected within 3 days after delivery and mature milk collected 30 days after delivery. The comparison of expression between the exosomes of human colostrum and mature milk was performed mainly on read count, and similar to cow milk, it was confirmed that the majority of the increased miRNAs had 2-4 times increased expression (Fig. 1A). In particular, it was confirmed that there were many miRNAs with increased expression of 2 to 4 times or less in the exosomes of human colostrum and that there were many miRNAs with increased expression of 4 times or more in the exosomes of mature human milk. The top 50 miRNAs most abundant in each exosome of human colostrum and mature milk are plotted in Fig. 1B. As a result, 37 out of 50 (58.7\% of each sample) were found in common, among which the top 6 miRNAs in exosomes of human colostrum were included. When comparing the ratios of the top 10 common miRNAs in exosomes of human colostrum and mature milk, it was confirmed that they existed at different ratios (Fig. 1C). In the case of exosomes of human colostrum, it was confirmed that hsa-miR-22-3p was the most abundant, and in the case of exosomes of mature milk, it was confirmed that hsa-miR-141$3 p$ was the most abundant. The identified miRNAs existed abundantly in human milk previous 
(A)

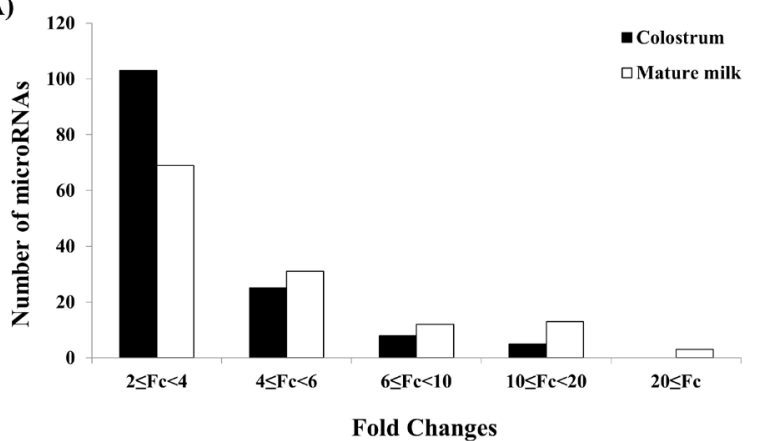

(C)

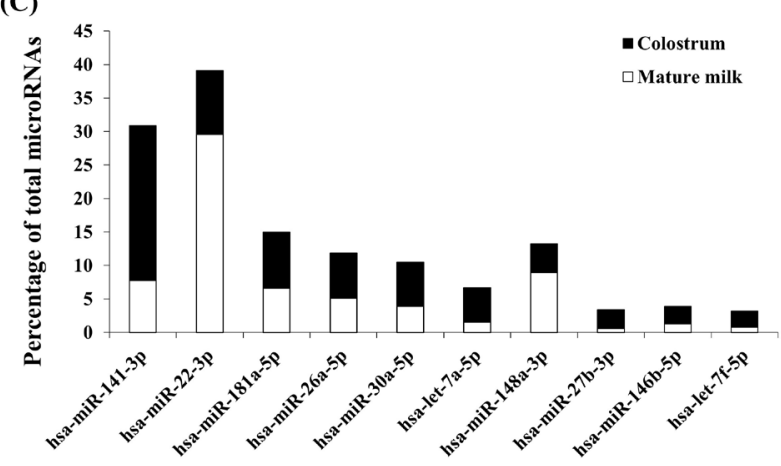

(B)

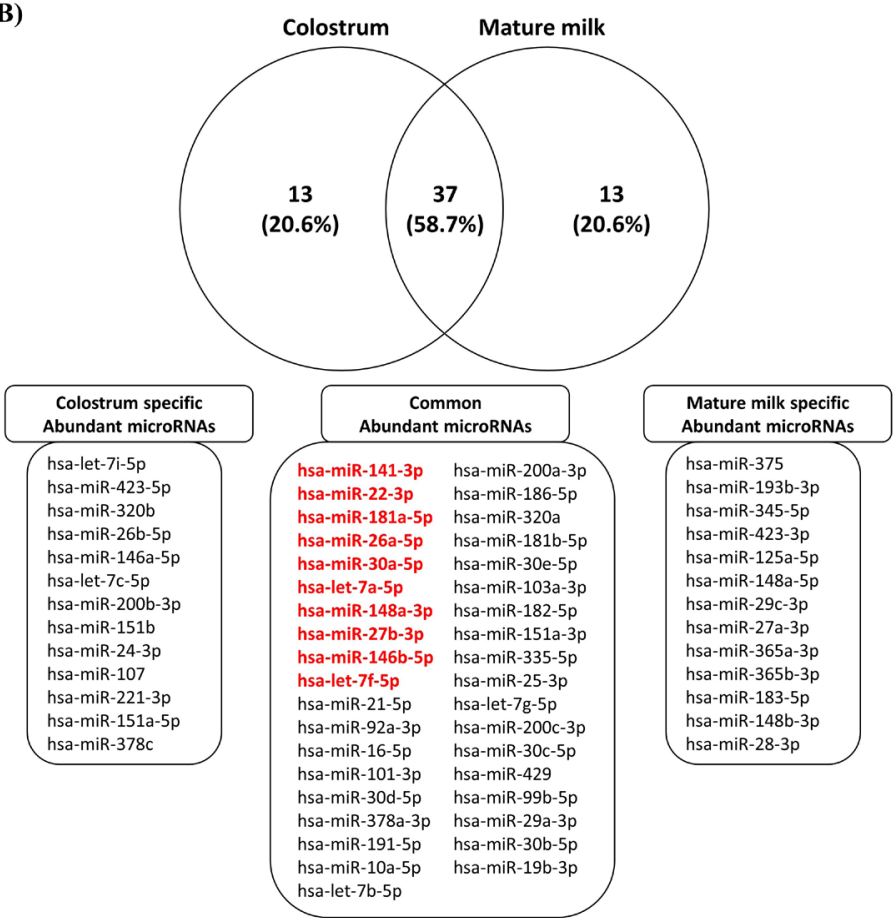

Fig. 1. MicroRNAs expressed in exosomes derived from human breast colostrum and mature milk. (A) Expression patterns of miRNAs expressed in human colostrum and mature milk. (B) Venn diagram depicting miRNAs expressed in human colostrum and mature milk. (C) Abundance profile of miRNAs in percentage (\%) for each miRNA of colostrum and mature milk in the top 10 miRNAs read (indicated with red) by next generation sequencing (NGS).

studies is consistent with results from previous studies [26,27]. Of the unique miRNAs most highly expressed in several studies [26,27], 9 miRNAs (except hsa-miR-27b-3p) were also found in the top 10 group in our study. This small but notable difference can be attributed to different ranking approaches, but it also implies a human-specific difference in the relative abundance of human milk exosome miRNAs. Additionally, based on results described previously [26-29], immune-related miRNAs were compared with those identified in our study. Among the top 50 miRNAs, 29 (58\%) and $25(50 \%)$ immune-related miRNAs were present in colostrum and mature milk, respectively. In particular, there were 23 (62.16\%) immune-related miRNAs in abundant miRNAs common to both colostrum and mature milk. These characterized miRNAs reinforce the idea that immune-associated miRNAs are abundant in breast milk exosomes, suggesting that more of these miRNAs could be detected.

\section{Comparison of miRNAs in exosomes from bovine colostrum and mature milk}

After collecting the colostrum and mature milk, it was quickly frozen at $-20^{\circ} \mathrm{C}$ and quickly transferred to the laboratory, and then exosomes were isolated and stored at $-80^{\circ} \mathrm{C}$. The miRNAs were isolated from exosomes isolated from bovine colostrum and mature milk. In the comparison of expression, miRNAs with 2- to 4-fold increased expression were the most abundant (Fig. 2A). In the exosomes of bovine colostrum, bta-miR-221 showed up to 48-fold expression compared to bovine mature milk, and in the exosomes of bovine mature milk, bta-miR-375 showed up to a 23-fold increase in expression. These miRNAs are thought to be some of the miRNAs showing functional differences between bovine colostrum and mature milk. The top 50 miRNAs, most abundantly present in bovine colostrum and mature milk exosomes, respectively, are plotted in Fig. 2B using 
(A)

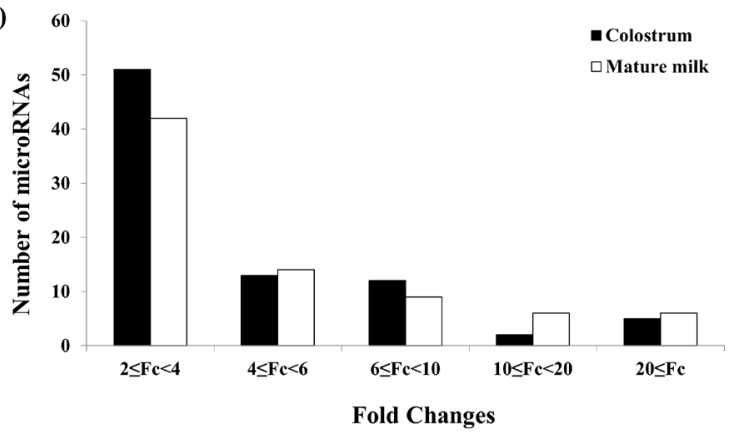

(C)

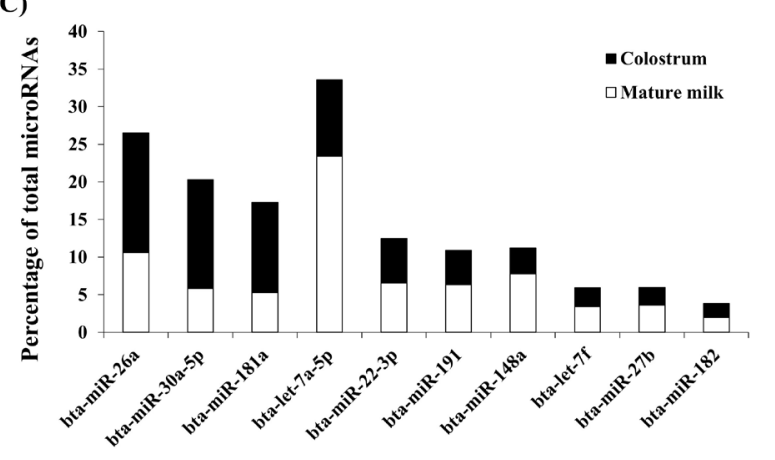

(B)

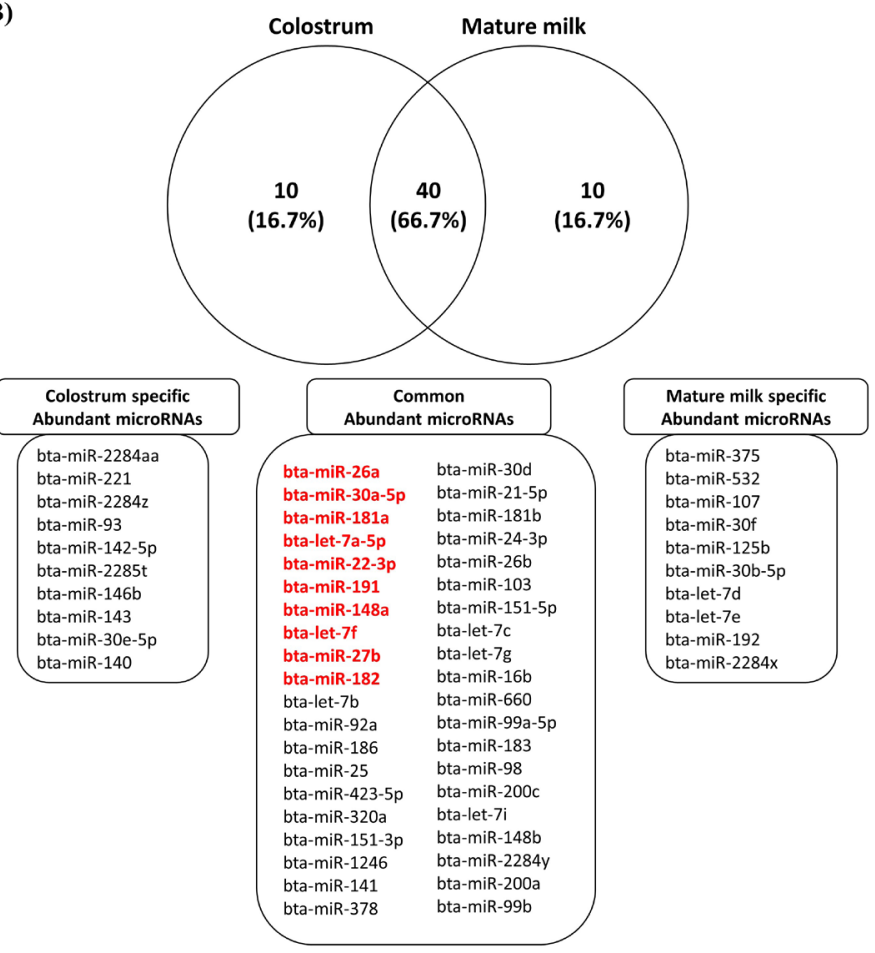

Fig. 2. MicroRNA expressed in exosomes from bovine colostrum and mature milk. (A) Expression patterns of miRNAs expressed in bovine colostrum and mature milk. (B) Venn diagram depicting miRNAs expressed in bovine colostrum and mature milk. (C) Abundance profile of miRNAs in percentage (\%) for each miRNA of colostrum and mature milk in the top 10 miRNAs read (indicated with red) by next generation sequencing (NGS).

a Venn diagram. When comparing the top miRNAs in the bovine colostrum and mature milk exosomes, there were around 40 miRNAs (66.7\% of each sample) in common, among which all of the top 10 miRNAs in the colostrum were included. In mature milk exosomes, 9 of them were included in the top 10. Colostrum-specific and mature milk-specific miRNAs were present in each of the 10 samples, and the corresponding lists are shown in Fig. 1B. The proportions in each sample of the top 10 miRNAs were different (Fig. 2C), indicating that there is potential for functional differences between bovine colostrum and mature milk. The overall ratio of the top 10 miRNAs was approximately $73 \%$ in bovine colostrum and approximately $75 \%$ in mature milk. There were 4 immune-related miRNAs (bta-miR-26a, bta-181a, bta-let-7a-5p, bta-miR-191, and bta-miR$27 \mathrm{~b}$ ) in the top 10 miRNAs among the common abundant miRNAs in colostrum and mature milk-derived exosomes $[15,30]$. In addition, among the many miRNAs common to colostrum and mature exosomes, bta-miR-26a, the most abundant miRNA in colostrum, and bta-let-7a-5p, the most abundant miRNA in mature milk, are immune-related miRNAs. Among the common top 10 miRNAs, 7 miRNAs (bta-miR-26a, bta-miR-181a, bta-let-7a-5p, bta-miR-191, bta-miR-148a, bta-let-7f, and bta-miR-27b) were abundant in milk, as shown in previous studies [15,30,31]. The shared 7 miRNAs might be associated with fundamental functions of bovine milk.

\section{Comparison of miRNAs in exosomes from caprine colostrum and mature milk}

Caprine milk was also profiled for miRNA using a small RNA sequencing method to compare colostrum collected within 3 days after delivery and mature milk collected 1 month after delivery. As a result of comparing the miRNA expression of caprine colostrum and mature milk exosomes, miRNAs with increased expression 2 to 4 times similar to cow and human milk exosomes 
(A)

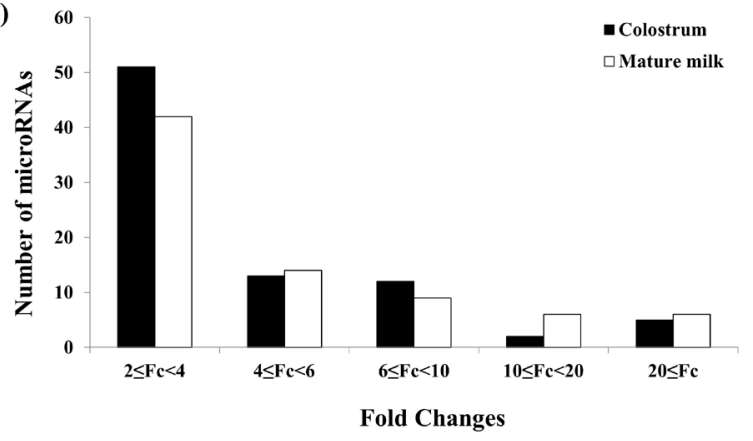

(C)

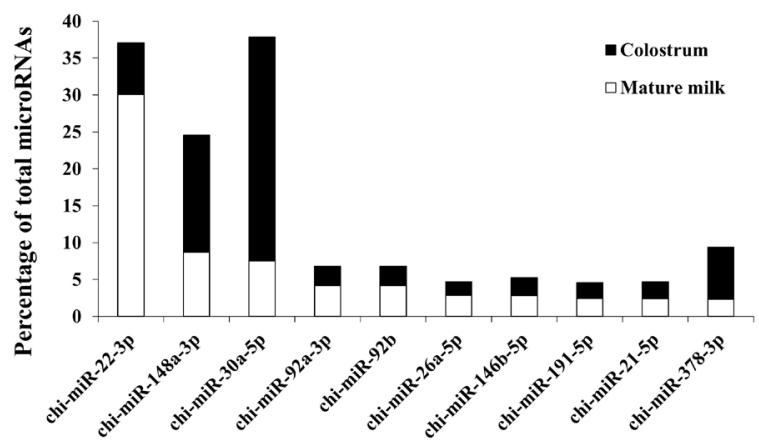

(B)

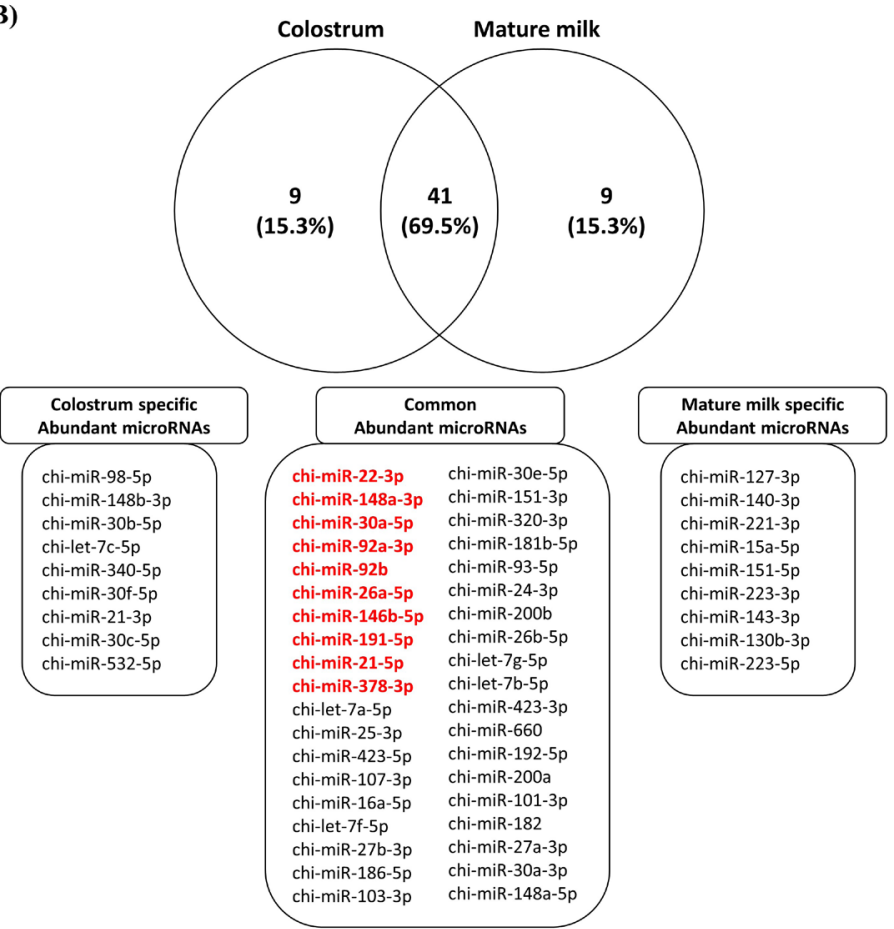

Fig. 3. MicroRNA expressed in exosomes from caprine colostrum and mature milk. (A) Expression patterns of miRNAs expressed in caprine colostrum and mature milk. (B) Venn diagram depicting miRNAs expressed in human colostrum and mature milk. (C) Abundance profile of miRNAs in percentage (\%) for each miRNA of colostrum and mature milk in the top 10 miRNAs read (indicated with red) by next generation sequencing (NGS).

accounted for the majority (Fig. 3A). The top 50 miRNAs, which are most abundant in caprine colostrum and mature milk exosomes, are plotted in a Venn diagram (Fig. 3B). As a result, among the top 50 miRNAs, 41 (69.5\% of each sample) were common, and among them, all of the top 10 miRNAs in the exosomes of caprine mature milk were included. When comparing the ratio of each sample of the top 10 miRNAs, it was confirmed that 3 (chi-miR-148a-3p, chi-miR-30a-5p and chi-miR-378-3p) of the top 10 miRNAs were more abundant in colostrum, and the remaining 7 were more abundant in mature milk (Fig. 3C). Of the top 10 miRNAs most highly expressed in caprine milk in previous studies [5,32], six miRNAs (chi-miR-21-5p, chi-miR-92a-3p, chi-miR92b, chi-miR148a-3p, chi-miR-146b-5p, and chi-miR-30a-5p) were included in the top 10 groups in our study. In addition, miR-148a is reported to be one of the most highly expressed miRNAs in caprine milk before and after pasteurization [5], and this study also found high levels of miR-148a. Interestingly, three of the four miRNAs (chi-miR-27b, chi-miR-103, and chi-miR-200a) reported to be involved in milk fat synthesis during caprine lactation were included in colostrum and mature milk [33]. In addition, two of the five miRNAs (chi-miR-146 and chi-miR-223) reported as immune-related miRNAs in caprine milk were identified in this study [34].

\section{Comparative analysis of miRNAs from human, bovine and caprine mature and colostrum milk-derived exosomes}

The top 10 miRNAs abundant in milk exosome-derived miRNAs for each species were investigated (Fig. 4). First, in colostrum exosome-derived miRNAs, a total of 4 miRNAs were common in humans, bovines and caprines (Fig. 4A). In addition, humans and bovines had a total of 8 miRNAs in common, and humans and caprines had 6 miRNAs in common. However, in the 
(A)

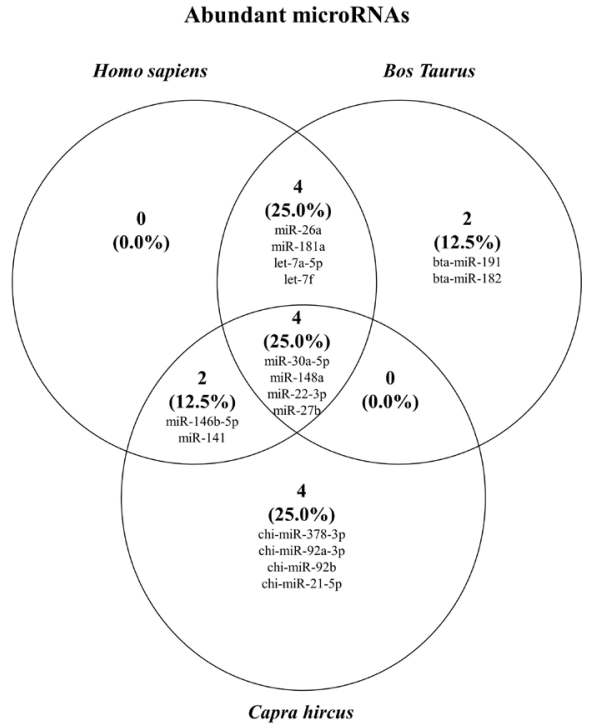

(B) Abundant microRNAs

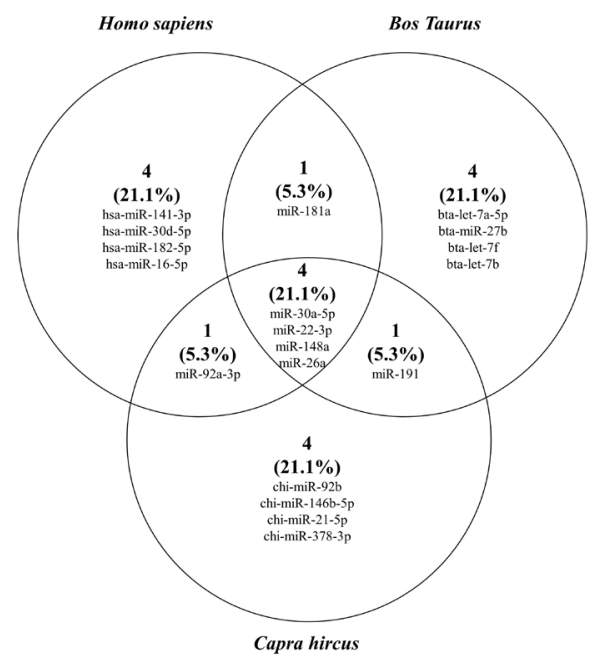

(C)

Common TOP 10 Abundant microRNAs

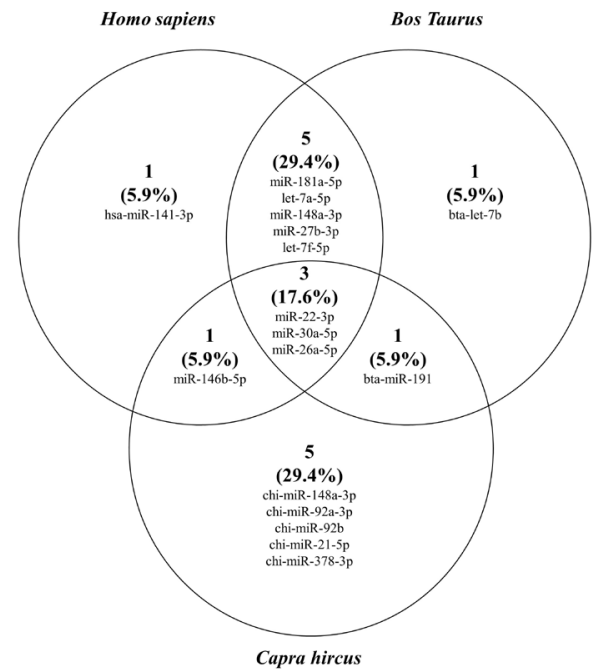

Fig. 4. Comparative analysis of exosome-derived microRNA in human, bovine and caprine colostrum and mature milk. (A) Venn diagram depicting the top 10 miRNAs expressed in human, bovine and caprine colostrum and mature milk. (B) Venn diagram depicting the top 10 miRNAs expressed in mature milk of humans, bovines and caprines. (C) Venn diagram depicting the top 10 miRNAs expressed in common in colostrum and mature milk from humans, bovines and caprines.

case of bovines and caprines, only four miRNAs existed in common with humans. In the case of mature milk, a total of 4 miRNAs were common in humans, bovines and caprines, similar to the colostrum-derived miRNAs (Fig. 4B). Humans and bovines, humans and caprines, and bovines and caprines each had 5 common miRNAs. Next, in miRNAs derived from both colostrum and mature milk, a total of 3 miRNAs existed in common from humans, bovines, and caprines. Interestingly, we found that milk-derived exosomes of bovines and humans have the most miRNAs ( 8 miRNAs) in common. These results propose that miRNAs in human and bovine milk are more similar than those in caprine milk.

Bovine and caprine milk are most widely applied as dietary dairy products, and it is important 
to determine their levels of miRNA expression, as it is a major component of infant formula [5]. Milk exosomal miRNAs are transferred from humans to humans as well as from livestock milk to influence on the expression of essential genes in target cells and tissues $[35,36]$. We showed in this study that highly expressed milk-derived miRNAs are primarily immune-related miRNAs [5,15,26-30,32]. In human colostrum, 6 miRNAs (miR-26a, miR-181a, let-7a-5p, miR-30a$5 p$, miR-22-3p, and miR-141) of the top 10 miRNAs are related to immunity, and 2 of them are common to cow and caprine colostrum. In human mature milk, 5 miRNAs (miR-141-3p, miR181a, miR-30a-5p, miR-22-3p, and miR-26a) in the top 10 were related to immunity, and 3 of them were common to cow and caprine milk. Interestingly, let-7a-5p, a miRNA that was relatively less expressed in human milk, was more abundant in cows and there was more miR-146b-5p in caprines. Among the miRNAs that are commonly expressed in human colostrum and mature milk, 7 miRNAs (including miR-22-3p, miR-30a-5p, and miR-26a-5p) of the top 10 miRNAs were associated with immunity, and 3 of them were identified as common between cattle and caprines. Interestingly, we indicated that the miRNAs that are highly expressed in human milk are similarly conserved across species. Milk is an abundant source of exosomal miRNAs that are evolutionarily conserved among mammals. Therefore, functional studies of other species of highly conserved exosomal miRNAs can be used to a variety of mammalian milk samples.

Taken together, we found that miR-30a-5p, miR-22-3p, and miR-26a are highly conserved in colostrum and mature milk in cows, caprines, and humans, suggesting their possible importance in neonatal growth. Our results provide new insights and resources for discovering the noble functionality of immune-associated miRNAs from dietary exosome [37] and for developing infant formulas taking into account the differences on miRNA expression in human, bovine and caprine milk as well as evaluating biological features as diagnostic biomarkers.

\section{REFERENCES}

1. Hoddinott P, Tappin D, Wright C. Breast feeding. BMJ. 2008;336:881-7. https://doi. org/10.1136/bmj.39521.566296.BE

2. Alsaweed M, Lai CT, Hartmann PE, Geddes DT, Kakulas F. Human milk miRNAs primarily originate from the mammary gland resulting in unique miRNA profiles of fractionated milk. Sci Rep. 2016;6:1-13. https://doi.org/10.1038/srep20680

3. Hassiotou F, Geddes DT. Programming of appetite control during breastfeeding as a preventative strategy against the obesity epidemic. J Hum Lact. 2014;30:136-42. https://doi. org/10.1177/0890334414526950

4. Newburg DS, Walker WA. Protection of the neonate by the innate immune system of developing gut and of human milk. Pediatr Res. 2007;61:2-8. https://doi.org/10.1203/01. pdr.0000250274.68571.18

5. Golan-Gerstl R, Elbaum Shiff Y, Moshayoff V, Schecter D, Leshkowitz D, Reif S. Characterization and biological function of milk-derived miRNAs. Mol Nutr Food Res. 2017;61:1700009. https://doi.org/10.1002/mnfr.201700009

6. Hata T, Murakami K, Nakatani H, Yamamoto Y, Matsuda T, Aoki N. Isolation of bovine milk-derived microvesicles carrying mRNAs and microRNAs. Biochem Biophys Res Commun. 2010;396:528-33. https://doi.org/10.1016/j.bbrc.2010.04.135

7. Izumi H, Kosaka N, Shimizu T, Sekine K, Ochiya T, Takase M. Bovine milk contains microRNA and messenger RNA that are stable under degradative conditions. J Dairy Sci. 2012;95:4831-41. https://doi.org/10.3168/jds.2012-5489

8. Özdemir S. Identification and comparison of exosomal microRNAs in the milk and co- 
lostrum of two different cow breeds. Gene. 2020;743:144609. https://doi.org/10.1016/ j.gene.2020.144609

9. Alsaweed M, Hartmann PE, Geddes DT, Kakulas F. MicroRNAs in breastmilk and the lactating breast: potential immunoprotectors and developmental regulators for the infant and the mother. Int J Environ Res Public Health. 2015;12:13981-4020. https://doi.org/10.3390/ ijerph121113981

10. Bartel DP. MicroRNAs: genomics, biogenesis, mechanism, and function. Cell. 2004;116:28197. https://doi.org/10.1016/S0092-8674(04)00045-5

11. Lee RC, Feinbaum RL, Ambros V. The C. elegans heterochronic gene lin-4 encodes small RNAs with antisense complementarity to lin-14. Cell. 1993;75:843-54. https://doi. org/10.1016/0092-8674(93)90529-Y

12. Alsaweed M, Hepworth AR, Lefèvre C, Hartmann PE, Geddes DT, Hassiotou F. Human milk microRNA and total RNA differ depending on milk fractionation. J Cell Biochem. 2015;116:2397-407. https://doi.org/10.1002/jcb.25207

13. Cheng L, Sun X, Scicluna BJ, Coleman BM, Hill AF. Characterization and deep sequencing analysis of exosomal and non-exosomal miRNA in human urine. Kidney Int. 2014;86:433-44. https://doi.org/10.1038/ki.2013.502

14. Li SP, Lin ZX, Jiang XY, Yu XY. Exosomal cargo-loading and synthetic exosome-mimics as potential therapeutic tools. Acta Pharmacol Sin. 2018;39:542-51. https://doi.org/10.1038/ aps.2017.178

15. Chen T, Xi QY, Ye RS, Cheng X, Qi QE, Wang SB, et al. Exploration of microRNAs in porcine milk exosomes. BMC Genomics. 2014;15:100. https://doi.org/10.1186/1471-2164-15100

16. Shandilya S, Rani P, Onteru SK, Singh D. Small interfering RNA in milk exosomes is resistant to digestion and crosses the intestinal barrier in vitro. J Agric Food Chem. 2017;65:9506-13. https://doi.org/10.1021/acs.jafc.7b03123

17. Pua HH, Happ HC, Gray CJ, Mar DJ, Chiou NT, Hesse LE, et al. Increased hematopoietic extracellular RNAs and vesicles in the lung during allergic airway responses. Cell Rep. 2019;26:933-44. https://doi.org/10.1016/j.celrep.2019.01.002

18. Xu H, Ling M, Xue J, Dai X, Sun Q, Chen C, et al. Exosomal microRNA-21 derived from bronchial epithelial cells is involved in aberrant epithelium-fibroblast cross-talk in COPD induced by cigarette smoking. Theranostics. 2018;8:5419-33. https://doi.org/10.7150/thno.27876

19. Admyre C, Johansson SM, Qazi KR, Filén JJ, Lahesmaa R, Norman M, et al. Exosomes with immune modulatory features are present in human breast milk. J Immunol. 2007;179:1969-78. https://doi.org/10.4049/jimmunol.179.3.1969

20. Reinhardt TA, Lippolis JD, Nonnecke BJ, Sacco RE. Bovine milk exosome proteome. J Proteomics. 2012;75:1486-92. https://doi.org/10.1016/j.jprot.2011.11.017

21. Li Z, Lan X, Guo W, Sun J, Huang Y, Wang J, et al. Comparative transcriptome profiling of dairy goat microRNAs from dry period and peak lactation mammary gland tissues. PLOS ONE. 2012;7:e52388. https://doi.org/10.1371/journal.pone.0052388

22. van Herwijnen MJC, Driedonks TAP, Snoek BL, Kroon AMT, Kleinjan M, Jorritsma R, et al. Abundantly present miRNAs in milk-derived extracellular vesicles are conserved between mammals. Front Nutr. 2018;5:81. https://doi.org/10.3389/fnut.2018.00081

23. Maburutse BE, Park MR, Oh S, Kim Y. Evaluation and characterization of milk-derived microvescicle isolated from bovine colostrum. Korean J Food Sci Anim Resour. 2017;37:654-62. https://doi.org/10.5851/kosfa.2017.37.5.654

24. Oh S, Park MR, Son SJ, Kim Y. Comparison of total RNA isolation methods for analysis of 
immune-related microRNAs in market milks. Korean J Food Sci Anim Resour. 2015;35:45965. https://doi.org/10.5851/kosfa.2015.35.4.459

25. Kozomara A, Griffiths-Jones S. miRBase: annotating high confidence microRNAs using deep sequencing data. Nucleic Acids Res. 2014;42:D68-73. https://doi.org/10.1093/nar/gkt1181

26. Liao Y, Du X, Li J, Lönnerdal B. Human milk exosomes and their microRNAs survive digestion in vitro and are taken up by human intestinal cells. Mol Nutr Food Res. 2017;61:1700082.

27. Zhou Q, Li M, Wang X, Li Q, Wang T, Zhu Q, et al. Immune-related microRNAs are abundant in breast milk exosomes. Int J Biol Sci. 2012;8:118-23. https://doi.org/10.7150/ijbs.8.118

28. Lindsay MA. microRNAs and the immune response. Trends Immunol. 2008;29:343-51. https://doi.org/10.1016/j.it.2008.04.004

29. Sonkoly E, Ståhle M, Pivarcsi A. MicroRNAs and immunity: novel players in the regulation of normal immune function and inflammation. Semin Cancer Biol. 2008;18:131-40. https:/doi. org/10.1016/j.semcancer.2008.01.005

30. Quan SY, Nan XM, Wang K, Zhao YG, Jiang LS, Yao JH, et al. Replacement of forage fiber with non-forage fiber sources in dairy cow diets changes milk extracellular vesicle-miRNA expression. Food Funct. 2020;11:2154-62. https://doi.org/10.1039/C9FO03097B

31. Kosaka N, Izumi H, Sekine K, Ochiya T. MicroRNA as a new immune-regulatory agent in breast milk. Silence. 2010;1:7. https://doi.org/10.1186/1758-907X-1-7

32. Hou J, An X, Song Y, Cao B, Yang H, Zhang Z, et al. Detection and comparison of microRNAs in the caprine mammary gland tissues of colostrum and common milk stages. BMC Genetics. 2017;18:1-8. https://doi.org/10.1186/s12863-017-0498-2

33. Lin X, Luo J, Zhang L, Zhu J. MicroRNAs synergistically regulate milk fat synthesis in mammary gland epithelial cells of dairy goats. Gene Expr J Liv Res. 2013;16:1-13. https://doi.org/1 $0.3727 / 105221613 X 13776146743262$

34. Huang Y. Expressional analysis of immune-related miRNAs in breast milk. Genet Mol Res. 2015;14:11371-6. https://doi.org/10.4238/2015.September.25.4

35. Baier SR, Nguyen C, Xie F, Wood JR, Zempleni J. MicroRNAs are absorbed in biologically meaningful amounts from nutritionally relevant doses of cow milk and affect gene expression in peripheral blood mononuclear cells, HEK-293 kidney cell cultures, and mouse livers. J Nutr. 2014;144:1495-500. https://doi.org/10.3945/jn.114.196436

36. Chen T, Xie MY, Sun JJ, Ye RS, Cheng X, Sun RP, et al. Porcine milk-derived exosomes promote proliferation of intestinal epithelial cells. Sci Rep. 2016;6:33862. https://doi.org/10.1038/ srep33862

37. Yun B, Maburutse BE, Kang M, Park MR, Park DJ, Kim Y, et al. Short communication: dietary bovine milk-derived exosomes improve bone health in an osteoporosis-induced mouse model.J Dairy Sci. 2020;103:7752-60. https://doi.org/10.3168/jds.2019-17501 